\title{
Curcumin Ameliorates the Impaired Insulin Signaling Involved in the Pathogenesis of Alzheimer's Disease in Rats
}

\author{
Tushar Kanti Das, ${ }^{\mathrm{a}, \mathrm{b}, *}$, Swarup Kumar Chakrabarti ${ }^{\mathrm{b}}$, Ihsan Nazurah Zulkipli ${ }^{\mathrm{a}}$ \\ and Mas R.W. Abdul Hamid ${ }^{\mathrm{a}}$ \\ ${ }^{\mathrm{a}}$ Universiti Brunei Darussalam, PAPRSB Institute of Health Sciences, Jalan Tungku Link, Gadong, Brunei \\ Darussalam, Brunei \\ ${ }^{\mathrm{b}}$ Institute of Reproductive Medicine, Salt Lake, Kolkata, India
}

Accepted 23 January 2019

\begin{abstract}
To date, dysregulation of the insulin signaling pathway in the brain has not been demonstrated unequivocally in Alzheimer's disease (AD). The purpose of the study was to examine the possible dysregulation of insulin signaling pathway in an AD rat model. Furthermore, the present study investigated the effect of Donepezil and Curcumin on insulin signaling, insulin, and glucose levels in AD rat brain. The rats were induced to develop AD by intraperitoneal administration of Scopolamine. We found that glucose levels in plasma and brain were decreased in AD rats, whereas the insulin levels was increased in plasma but decreased in brain in AD rats. In addition, insulin signaling proteins IR- $\beta$, IGF- 1 , IRS- 1 , IRS- 2 p-Akt (Ser473), and Akt were markedly reduced in the AD rats. Furthermore, GLUT3 and GLUT4 levels in the brain were markedly reduced in AD rats. All these data were compared to Saline-treated control rats. Curcumin significantly increased glucose levels in plasma and in brain. However, insulin levels was decreased in plasma and was increased in AD rats' brain. Moreover, GLUT3 and GLUT4 levels were significantly increased in Curcumin-treated AD rats. All these data were compared to Scopolamine-induced AD rats. Thus amelioration of impaired insulin signaling and improved glucose regulation in AD rats by Curcumin may be beneficial in the management of AD.
\end{abstract}

Keywords: Alzheimer's disease, curcumin, donepezil, glucose receptors, insulin signaling

\section{INTRODUCTION}

Alzheimer's disease (AD) is the most devastating neurodegenerative disease among older people $(\geq 65$ years) that ultimately causes death of the affected individuals. $\mathrm{AD}$ is a major global health problem that results in significant burden on the patients due to the high cost of managing AD [1]. Neuropathologically,

\footnotetext{
*Correspondence to: Tushar Kanti Das, PhD, Institute of Reproductive Medicine, Salt Lake, Kolkata, India. Tel.: +91 7430877 739; E-mail: dtushar30@gmail.com.
}

$\mathrm{AD}$ is characterized by brain atropy, loss of neurons, synaptic dysfunction, extracellular accumulation of amyloid- $\beta(A \beta)$, and intracellular neurofibrillary tangles (NFT) composed of hyperphosphorylated tau [2, 3]. Despite indistinguishable clinical dementia symptoms, less than $1 \%$ familial early-onset $\mathrm{AD}$ is caused by missense mutations in three genes of presenilin-1, presenilin-2, and amyloid precursor protein [4]. However, most $\mathrm{AD}$ cases are sporadic and are believed to result from multiple etiologic factors including genetic susceptibility (such as ApoE4 allele), aging, type 2 diabetes, and metabolic factors $[5,6]$. 
Defective glucose utilization [7-9] or hypometabolism of glucose in the brain may precede the onset of cognitive deficits [10-12]. Furthermore, hyperinsulinemia is associated with higher risk of $\mathrm{AD}$ and decline in memory [7, 8, 11]. Peripheral glucose metabolism is primarily regulated by insulin. Therefore, hyperinsulinemia associated with insulin resistance can affect insulin signaling that controls brain glucose metabolism and facilitates the neuronal development and functions in learning and memory [13]. It is hypothesized that possible dysfunction of insulin signaling is linked with AD pathogenesis [14-17]. Although, how the impaired insulin signaling contributes to the $\mathrm{AD}$ pathogenesis is not fully understood.

Numerous research groups [18-21] have provided robust evidence regarding the supporting evidences the use of Scopolamine in AD animal model. Scopolamine, a muscarinic cholinergic receptor antagonist, has been commonly used for the study of cognitive deficiency in experimental animals. Intraperitoneal injection (i.p.) of Scopolamine into animals causes blockage of the cholinergic neurotransmission that leads to cholinergic dysfunction and impaired cognition in animals [18-21]. Bihaqi et al. found that the expression of tau and the amyloid- $\beta$ protein precursor (A $\beta P P)$ were increased in rat brain [20]. Scopolamine-induced dementia model is the most appropriate dementia model to reveal AD-related cognitive impairment profile [21]. Donepezil, a wellknown pharmacological drug used for the treatment of $\mathrm{AD}$, acts as an acetylcholinesterase inhibitor, reduces the hydrolysis of acetylcholine, and improves AD pathogenesis. Throughout this study, Donepezil was used as a reference drug. Curcumin, a natural compound derived from plant, is also known for its protective effect on AD pathogenesis. It is known that Curcumin has various properties such as antiinflammatory, anti-oxidant, and scavenger of free radicals [20]. Importantly, curcumin has also been shown to have beneficial effects in various neurodegenerative disease models [23-26]. However, to date, there is very little information about any effects of Donepezil and Curcumin on insulin signaling, glucose, and insulin levels in AD rats.

Thus, in this study, we investigated the effects of Donepezil and Curcumin on the mediators of insulin signaling such as IR- $\beta$, IGF-I, IRS-1/2, PI3K, p-Akt (Ser473), and Akt and also on the levels of key glucose transporters such as GLUT3 and GLUT4 in a rat model of sporadic AD, which was generated by i.p injection of Scopolamine. In addition, the effects of
Donepezil and Curcumin on glucose and insulin levels in both plasma and in the brain of AD rats were evaluated. We found that glucose levels in plasma and brain were decreased in $\mathrm{AD}$ rats. Whereas, the insulin levels was increased in plasma but decreased in brain in $\mathrm{AD}$ rats. In addition, insulin signaling proteins IR- $\beta$, IGF-1, IRS-1, IRS-2 p-Akt (Ser473), and Akt were markedly reduced in the AD rats. Furthermore, GLUT3 and GLUT4 levels in the brain were markedly reduced in AD rats. All these data were compared to Saline-treated control rats. Curcumin significantly increased glucose levels in plasma and in brain. However, insulin levels was decreased in plasma and was increased in AD rat brain. Moreover, GLUT3 and GLUT4 levels were significantly increased in Curcumin-treated AD rats. All these data were compared to Scopolamine-induced AD rats.

\section{MATERIAL AND METHODS}

\section{Materials}

Scopolamine, Donepezil, and Curcumin were purchased from Sigma Aldrich (St. Louis, MO, USA). Protease inhibitor tablets were purchased from Roche Applied Science (Mannheim, Germany). Amplex Red Glucose/glucose Oxidase Assay Kit were purchased from Invitrogen (Carlsbad, CA, USA) and ELISA kit for Insulin were purchased from CloudClone Corp (Houston, TX, USA). Antibodies against IR- $\beta$, IGF-I, IRS-1, IRS-2, Akt (pan), p-Akt (Ser 473), and GLUT4 were purchased from Cell Signaling Technology (Danvers, MA, USA). p-PI3K (p85) was purchased from Santa Cruz Biotechnology (Dallas, TX, USA). Antibodies against GLUT3 and $\beta$-Actin were purchased from Upstate Biotechnology (Lake Placid, NY, USA) and Sigma Aldrich (St. Louis, MO, USA) respectively. Immunohistochemistry kit (R.T.U. Vectastain Universal Elite $\mathrm{ABC}$ Kit and DAB kit) was purchased from Vector Laboratories (Burlingame, CA, USA). Pierce ${ }^{R}$ BCA Protein Assay Kit and ECL advanced reagent kit were purchased from Thermo Fisher Scientific (Waltham, MA, USA) and Bio-Rad (Hercules, CA, USA) respectively. Other analytical grade reagents were purchased from Merck (Kenilworth, NJ, USA).

\section{Animals}

24 male Sprague Dawley rats aged 60-70 days and weighted $300 \pm 20 \mathrm{~g}$ provided by PAPRSB Institute of Health Sciences, UBD were utilized. The 
Table 1

Experimental design and drug doses in different groups of rats

\begin{tabular}{lll}
\hline Group No. & Name of groups & Compound and drug administered \\
\hline 1 & Control & Saline $(0.9 \%$ w $/ \mathrm{v})$ i.p. \\
2 & Disease Control & Scopolamine $(2.50 \mathrm{mg} / \mathrm{kg})$ i.p. \\
3 & Standard Treatment & Scopolamine $(2.50 \mathrm{mg} / \mathrm{kg})$ i.p. + Donepezil $(5 \mathrm{mg} / \mathrm{kg})$ oral \\
4 & Test group & Scopolamine $(2.50 \mathrm{mg} / \mathrm{kg})$ i.p + Curcumin $(80 \mathrm{mg} / \mathrm{kg})$ oral \\
\hline
\end{tabular}

rats were provided standardized rat food (Specialty Feeds, Western Australia) and drinking water, and were housed in temperature-controlled cages (12-h light/dark cycle, room temperature $26^{\circ} \mathrm{C} \pm 2^{\circ} \mathrm{C}$ ). The experiments were performed according to standard guideline approved by PAPRSB, Institutes of Health Sciences Research Ethic Committee and Universiti Brunei Darussalam Research Ethic Committee.

\section{Study design}

The rats were randomly divided into four groups as follows; (a) Control (saline-treated), (b) Scopolamine-induced, (c) Scopolamine+Donepeziltreated, and (d) Scopolamine+Curcumin-treated. Control group was administered with $0.9 \%$ saline (w/v) for 28 consecutive days. Scopolamine $(2.5 \mathrm{mg} / \mathrm{kg})$, as a disease inducer, was administered to all the groups through i.p route. The treatment groups were given Donepezil $(5 \mathrm{mg} / \mathrm{kg})$ and Curcumin $(80 \mathrm{mg} / \mathrm{kg})$ by oral gavage for 28 consecutive days (Table 1). Although oral gavage was as invasive procedure, however we did not observe any signs of suffering in animal in the study.

\section{Preparation of blood plasma}

After 28 days of either Scopolamine or Saline treatment, rats were anesthetized using carbon dioxide in a closed chamber and then sacrificed by decapitation using guillotine. $5 \mathrm{ml}$ blood was collected in anticoagulant heparin tube and it was fractionated by centrifugation at $3000 \times \mathrm{g}$ for $15 \mathrm{~min}$. Supernatants (plasma) were collected and stored frozen at $-80^{\circ} \mathrm{C}$ until further use.

\section{Brain tissue preparation and processing}

After 28 days of either Scopolamine or Saline treatment, rats were anesthetized using carbon dioxide in a closed chamber and then sacrificed by decapitation using guillotine, dissected and the brains were quickly collected and washed three times in chilled $1 \mathrm{x}$ phosphate buffered saline (PBS) (pH 7.4) (Bio-Rad).
Each group consists of six brains in which two brains were used for immunohistochemistry (IHC) and the remaining four brains were used for immunoblotting.

\section{Immunohistochemistry}

Brains from each group were washed in 1X PBS, and were subsequently used in tissue processing. Each brain from rat was cut into smaller pieces using a tissue slicer and then processed using an automated tissue processor (Leica ASP 300 S Tissue Processor, Leica Microsystem, USA), embedded in paraffin wax and then sectioned using a microtome (Medite, Medizintechnik, Germany) into 4- $\mu \mathrm{m}$ thickness. Brain sections from different groups of rats ( $n=2$ at each time point for each rat) were used for IHC using R.T.U Vectastain Universal Elite ${ }^{\circledR}$ ABC Kit (Vector Laboratories). Deparaffinised brain tissue sections were heat-induced using sodium citrate buffer $(10 \mathrm{mM}$ sodium citrate, $0.05 \%$ tween $20, \mathrm{pH}$ 6.0) and quenched with $0.3 \%$ hydrogen peroxide, blocked with $2.5 \%$ normal horse serum. Primary antibodies such as [IR- $\beta$, p-Akt (Ser 473), Akt and GLUT4] were incubated at $4{ }^{\circ} \mathrm{C}$ for whole night. Subsequent procedure was done according to manufacturer's direction (Vector Universal elite ${ }^{\circledR}$ ABC kits, Vector Laboratories). Finally, tissues were counterstained with Hematoxylin, dehydrated and mounted. These sections were observed using a light microscope with lens objective 40x (Olympus, USA).

\section{Brain homogenate preparation}

The remaining four brains from each group were used for immunoblotting. Each brain with known weight was snapped frozen in liquid nitrogen, homogenized in cell lysis buffer (1X) (Cell Signalling Technology) containing protease inhibitor tablet (Roche) at 3 pulses for $10 \mathrm{~s}$ each, with a $5 \mathrm{~s}$ interval on ice. Tissue lysates were diluted 10 -fold in $1 \mathrm{X}$ cell lysis buffer and were sonicated to ensure complete lysis. The crude brain homogenates were stored at $-80^{\circ} \mathrm{C}$ and the protein concentrations were 
determined by using Pierce ${ }^{\mathrm{R}}$ BCA Protein Assay Kit (Thermo Fisher Scientific).

\section{Measurements of glucose and insulin levels}

Glucose levels in plasma and in brain were measured using Amplex Red Glucose/glucose Oxidase Assay Kit (Invitrogen) and the insulin concentrations were measured in plasma and in brain using ELISA (Cloud-Clone Corp) following the instructions provided by the manufacturer. Absorbance was measured at $560 \mathrm{~nm}$ and $450 \mathrm{~nm}$ (BioTek Instruments, USA) respectively.

\section{Immunoblotting}

$50 \mu \mathrm{g}$ proteins were separated on 10-12.5\% SDSpolyacrylamide gel and transferred to nitrocellulose membranes (Amersham Bioscience, Piscataway, NJ, USA), blocked for $1 \mathrm{~h}$ at room temperature with $4-5 \%$ BSA in T-TBS, incubated at $4{ }^{\circ} \mathrm{C}$ overnight with respective primary antibodies, followed by incubation in horseradish peroxidase (HRP) conjugated secondary antibodies. Finally, the reactive protein bands were visualized using chemiluminescence detection reagent (Clarity $^{\mathrm{TM}}$ Western ECL Substrate, Bio-Rad) and signals were captured and visualized using VersaDoc Imaging system (Bio-
Rad). Pre-stained Precision Plus ${ }^{\mathrm{TM}}$ protein standards (Bio-Rad) was used to estimate the apparent molecular weight of the protein bands. $\beta$-actin was used as a control to ensure equal amount of protein loading.

\section{Statistical analysis}

Data were expressed as mean \pm S.E. and analyzed using SPSS 20.0 statistical software (SPSS Inc., Chicago, IL, USA). The post-hoc test were used after ANOVA analysis. A value of $p<0.05$ was considered significant.

\section{RESULTS}

\section{Lower levels of plasma glucose and brain glucose in $A D$ rats and curcumin treatment significantly increased glucose levels}

Glucose contents in the plasma were measured in all groups of rats and showed in Fig. 1. The glucose concentration in the plasma $(n=6)$ was $142.17 \pm 2.58 \mathrm{mg} / \mathrm{dL}$ in Saline-treated control group and $111.77 \pm 1.68 \mathrm{mg} / \mathrm{dL}$ in the Scopolamineinduced AD rats. Donepezil-treatment in AD rats increased glucose levels to $126.04 \pm 2.87 \mathrm{mg} / \mathrm{dL}$ compared to Scopolamine-induced AD rats. Furthermore, Curcumin-treatment in $\mathrm{AD}$ rats also increased
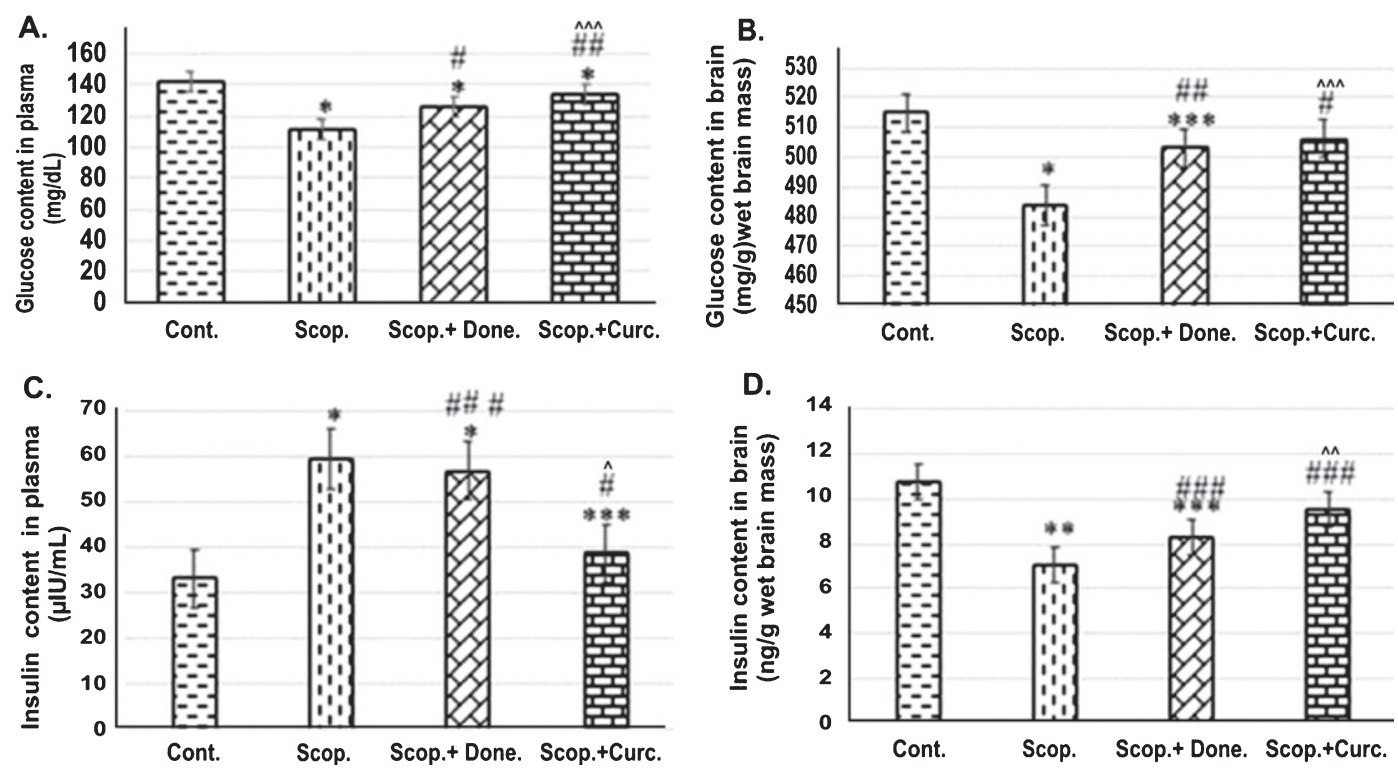

Fig. 1. ELISA analysis of (I) glucose and (II) insulin content in (A) the plasma $(n=8)(\mathrm{B})$ the brain $(n=4)$ at each time point for each rat line. Each value represents the mean \pm S.E. ${ }^{*} p<0.001,{ }^{*} p<<0.01$, and $* * * p<0.05$ compared with control group; ${ }^{\#} p<0.001$, ${ }^{\# \#} p<0.01$, and

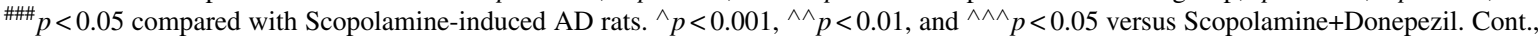
Control; Scop., Scopolamine; Done., Donepezil; Curc., Curcumin. 
glucose concentration to $133.94 \pm 2.81 \mathrm{mg} / \mathrm{dL}$. (Fig. 1A) compared to Scopolamine-induced AD rats. Taken together, it was found that the glucose levels was decreased 1.27 -fold $(p<0.001)$ in the Scopolamine-induced AD rats compared to Salinetreated control rats. Treatment with Donepezil and Curcumin in AD rats increased glucose levels by 1.13 -fold $(p<0.001)$ and 1.2 -fold $(p<0.001)$, respectively, compared to those in Scopolamine-induced $\mathrm{AD}$ rats.

Furthermore, glucose concentration in the brain was measured in all groups of rats. Glucose concentration in the brain lysate was $515 \pm 4.22 \mathrm{mg} / \mathrm{g}$ wet brain mass in the Saline-treated control group and $483.76 \pm 9.69 \mathrm{mg} / \mathrm{g}$ wet brain mass in Scopolamineinduced AD group respectively. In Donepezil-treated and Curcumin-treated $\mathrm{AD}$ rats, glucose concentration was found to be $503.02 \pm 5.10 \mathrm{mg} / \mathrm{g}$ wet brain mass and $506.32 \pm 2.26 \mathrm{mg} / \mathrm{g}$ wet brain weight (Fig. 1B) respectively. Taken together, it was found that glucose level was decreased 1.52 -fold $(p<0.001)$ in Scopolamine-induced AD rats compared to Salinetreated control rats. Treatment with Donepezil and Curcumin separately in AD rats increased glucose levels 1.04-fold $(p<0.01)$ and 1.4-fold $(p<0.001)$ respectively compared to those in Scopolamineinduced $\mathrm{AD}$ rats.

\section{Higher levels of plasma insulin and lower levels of brain insulin in $A D$ rat, curcumin treatment significantly reversed the insulin levels}

Plasma insulin levels were measured in all groups of rats. Insulin concentration was found to be $33.11 \pm 1.16 \mu \mathrm{IU} / \mathrm{mL}$ in Saline-treated control group in comparison to $59.38 \pm 3.56 \mu \mathrm{IU} / \mathrm{mL}$ in Scopolamine-induced AD rats. In Donepezil-treated $\mathrm{AD}$ rats, the insulin concentration was found to be $56.94 \pm 0.44 \mu \mathrm{IU} / \mathrm{mL}$. Interestingly, Curcumin treatment in $\mathrm{AD}$ rats markedly lowered insulin levels to $38.74 \pm 0.44 \mu \mathrm{IU} / \mathrm{mL}$ compared to Scopolaminetreated $\mathrm{AD}$ rats. Thus, plasma insulin level was 1.79 -fold higher $(p<0.001)$ in Scopolamine-induced AD rats compared to Saline-treated control rats. Treatment with Donepezil and Curcumin separately in AD rats decreased insulin level 1.04-fold $(p<0.05)$ and 1.53 -fold $(p<0.001)$, respectively, compared to those in Scopolamine-induced AD rats (Fig. 1C).

Furthermore, insulin levels in the brain were measured in all groups of rats. The insulin level in the brain was $10.78 \pm 1.64 \mathrm{ng} / \mathrm{g}$ wet brain mass and $7.08 \pm 1.25 \mathrm{ng} / \mathrm{g}$ wet brain mass in Saline-treated and Scopolamine-induced AD rat group respectively. In Donepezil-treated AD rats, the insulin level was at $8.3 \pm 1.86 \mathrm{ng} / \mathrm{g}$ wet brain mass. In addition, Curcumin- treatment in AD rats increased insulin levels to $9.53 \pm 1.25 \mathrm{ng} / \mathrm{g}$ (Fig. 1D) compared to Scopolamine-induced AD rats. Therefore, it was found that insulin level was 1.52 -fold $(p<0.01)$ decreased in AD rats compared to Saline-treated control rats. Treatment with Donepezil and Curcumin separately in $\mathrm{AD}$ rats increased insulin level 1.17 -fold $(p<0.01)$ and 1.42 -fold $(p<0.05)$, respectively, compared to those in Scopolamine-induced $\mathrm{AD}$ rats.

\section{Reduced number of immunostained cells positive for insulin signaling proteins and glucose transporter in $A D$ rat brain and curcumin reversed the effects}

Immunohistochemistry was used to detect the presence of insulin signaling proteins and glucose transporter in the brain cortex of the rats. There was a reduced number of the immunostained cells for (i) IR- $\beta$, (ii) Akt, (iii) Phospho-Akt (Ser473), and (iv) GLUT4 in AD rats compared to Saline-treated control rats. Treatment with either Donepezil or Curcumin increased numbers of positive insulin signaling proteins such as IR- $\beta$, Akt, Phospho-Akt (Ser473), and GLUT4 immunopositive cells in AD rats (Fig. 2).

\section{Lowered levels of upstream insulin signaling proteins in $A D$ rat brain and curcumin significantly reversed it}

To examine the expression of major insulin receptor in the brain involved in insulin signaling pathway, we investigated the protein levels of insulin receptor $\beta$-subunit (IR- $\beta$ ) (Fig. 3a, a') and IGF1 receptor (IGF-1) (Fig. 3b, b') in AD-rat brain homogenates by immunoblotting. We found a significant decrease in the levels of IR- $\beta$ (0.72-fold, $p<0.001)$ in AD rats compared to the Saline-treated rats. Densitometric analysis showed increased levels of IR- $\beta$ in both Donepezil (1.06-fold, $p=0.074$ ) and Curcumin-treated AD (1.25-fold, $p<0.001)$ rats upon treatment with separately Donepezil (1.06-fold, $p=0.074)$ and Curcumin (1.25-fold, $p<0.001)$ compared to Scopolamine-induced rats. Furthermore, in AD rats, the IGF-1 level was decreased (0.62-fold, $p<0.001)$ compared to Saline-treated rats. Donepezil and Curcumin treatment separately on $\mathrm{AD}$ rats decreased $(0.78$-fold, $p<0.01)$ and increased (1.14- 
Cont.

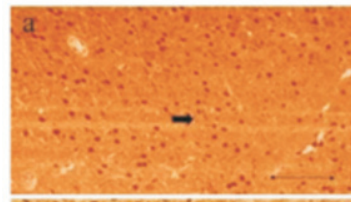

IR- $\beta$

Akt

GLUT4
Scop.
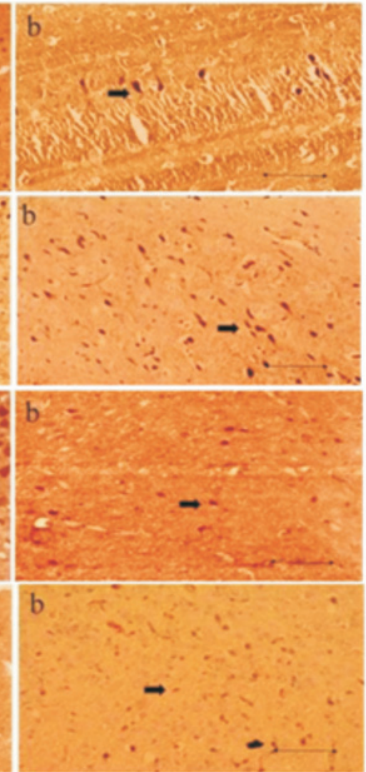

Scop. + Done.
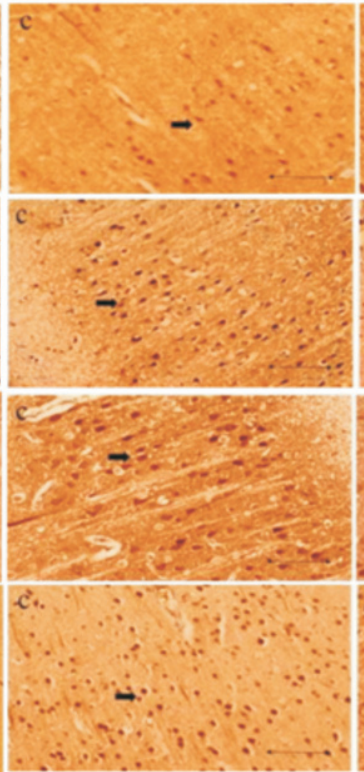

Scop. + Curc

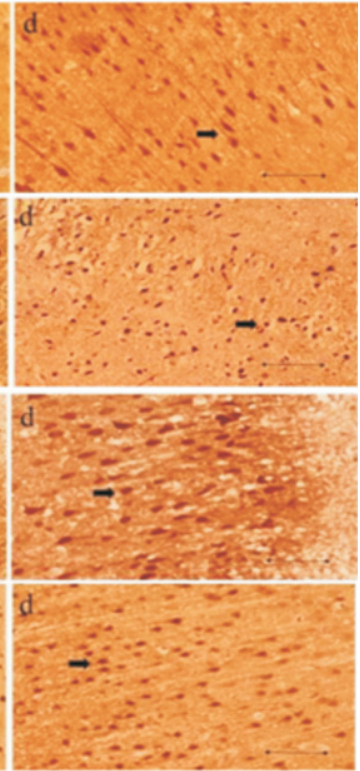

Fig. 2. Immunohistochemistry of (i) IR- $\beta$, (ii) Akt, (iii) p-Akt (Ser 473), and (iv) GLUT4 in the brain sections (cortex) of several groups of rats. The numbers of immunopositive cells stained with IR- $\beta$, Akt, p-Akt (Ser 473) and GLUT4 antibody were decreased in Scopolamineinduced $\mathrm{AD}$ rat brain and were increased in Donepezil- and Curcumin-treated $\mathrm{AD}$ rats. The assay was performed in triplicate. Lens objective: 40x. Scale bar represents $1 \mu$. Cont., control; Scop., Scopolamine; Done., Donepezil; Curc., Curcumin.

fold, $p<0.05)$ IGF-1 levels respectively compared to Scopolamine-induced AD rats (Fig. 3).

We next investigated the expressions of insulin receptor substrate 1(IRS-1) (Fig. 3c, c') and IRS-2 (Fig. 3d, d') in rat brain. It was found that the levels of IRS-1 (0.74-fold, $p<0.001)$ and IRS-2 (0.62-fold, $p<0.001)$ were decreased in AD rats compared to Saline-treated rats. Donepezil treatment in AD rats lowered the levels of IRS-1 (0.77-fold, $p<0.001)$ and IRS-2 (0.82-fold, $p<0.01)$ respectively, as compared to Scopolamine-induced rats. On the contrary, Curcumin treatment in $\mathrm{AD}$ rats augmented the levels of both IRS-1 (1.22-fold, $p<0.001)$ (Fig. 3c, c') and IRS-2 (1.31-fold, $p<0.01$ ) (Fig. 3d, d') as compared to those in Scopolamine-induced rats.

\section{Reduced levels of downstream insulin signaling proteins in $A D$ rat brain, curcumin significantly reversed it}

We examined quantitatively the expression of $\mathrm{p}$ PI3K (p85) (Fig. 3e, e') in the rat brains. In AD rats, the levels of p-PI3K (p85) was significantly decreased (0.59-fold, $p<0.001)$ compared to Saline-treated control rats. Donepezil and Curcumin- treatment separately in AD rats increased p-PI3K (p85) levels (1.02-fold, $p<0.05$ and 1.03-fold, $p<0.05$ ), respectively, compared to Scopolamine-induced rats (Fig. 3e, e'). We further examined the levels of pAkt (Ser473) (Fig. 3f, f') and Akt (Fig. 3g, g') in rat brains from several groups. In AD rats, the levels of p-Akt (Ser473) $(0.57$-fold, $p<0.001)$ and Akt $(0.66-$ fold, $p<0.001)$ levels were significantly decreased as compared to Saline-treated control rats. Donepezil and Curcumin-treatment in $\mathrm{AD}$ rats increased levels of p-Akt (Ser473) (1.39-fold, $p<0.001)$, Akt (1.19fold, $p<0.001)$ and (1.41-fold, $p<0.001)$ (Fig. 3f, f'), (1.09-fold, $p<0.01$ ) (Fig. 3g, g') respectively compared to Scopolamine-induced rats.

\section{Lowered levels of glucose transporters in $A D$ rat brain, curcumin significantly reversed it}

To investigate if there was any alteration in the levels of glucose transporters among various rat groups, we examined expression of the two key glucose transporters (GLUT3 and GLUT4) in the rat brains quantitatively using immunoblotting (Fig. 3). We observed that the levels of both GLUT3 (0.71fold, $p<0.001$ ) (Fig. 3h, h') and GLUT4 (0.87-fold, $p<0.001$ ) (Fig. 3i, i') were markedly decreased in $\mathrm{AD}$ rats compared to Saline-treated rats. Treatment 
L1 L2 L3 L4

(a) IR- $\beta$

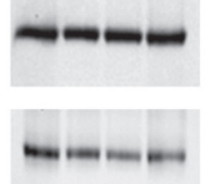

$95 \mathrm{kDa}$

(b) IGF-1

$95 \mathrm{kDa}$

(c) IRS-1

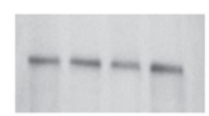

$180 \mathrm{kDa}$

(d) IRS-2

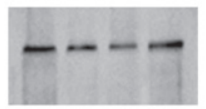

$185 \mathrm{kDa}$

(a')

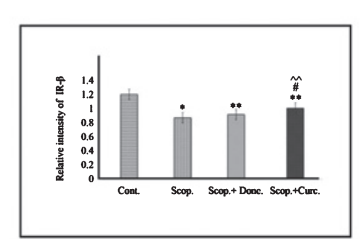

(b')

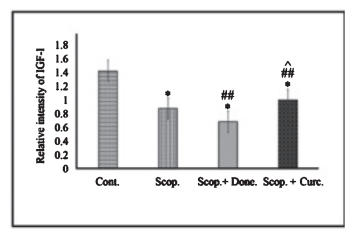

( $\left.c^{\prime}\right)$

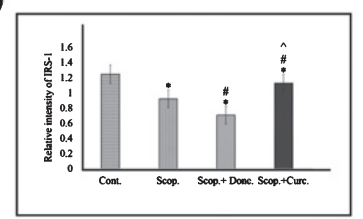

(e')

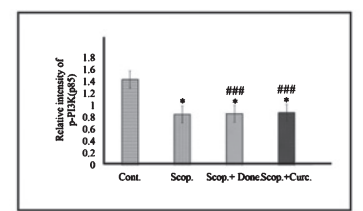

(g')

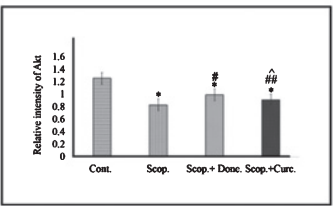

(h')

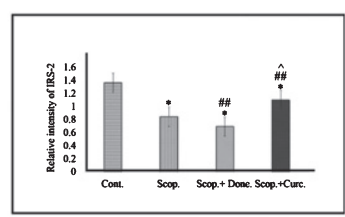

(f')
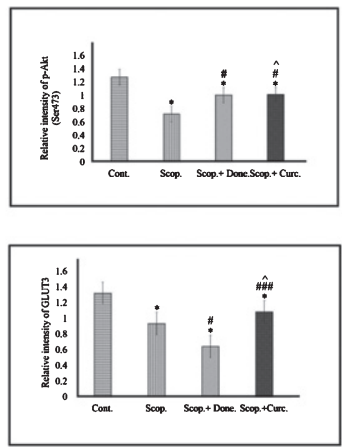

(g) Akt

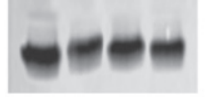

60 kDa

$\beta$-Actin

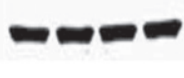

(h) GLUT3

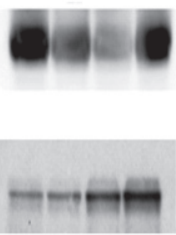

(i) GLUT4

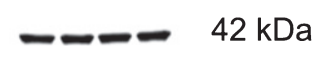

$\beta$-Actin
42 kDa

60 kDa

50 kDa

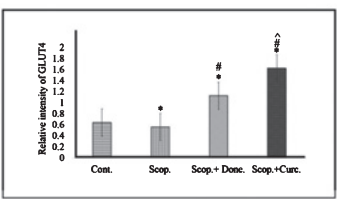

Fig. 3. Immunoblotting and densitometry analysis of (a) IR- $\beta$, (b) IGF-I, (c) IRS-1, (d) IRS-2, (e) p-PI3K(p85), (f) p-Akt (Ser473), (g)Akt, (h) GLUT3, and (i) GLUT4 in brain lysates. Analysis was performed using NIH ImageJ software and the values obtained for the individual protein target for each brain sample were normalized against $\beta$-actin from the same brain sample. The assay was performed in triplicate and ANOVA was used to analyze the differences among groups. Each value represents mean \pm S.E. for individual rat brain sample $(n=4)$. Here L1 = Control, L2 = Scopolamine-induced, L3 = Scopolamine+Donepezil and L4 = Scopolamine+ Curcumin group. $* p<0.001, * * p<0.01$, and ${ }^{* * *} p<0.05$ versus Saline-treated control; ${ }^{\#} p<0.001,{ }^{\# \#} p<0.01$, and ${ }^{\# \# \#} p<0.05$ versus Scopolamine-induced; ${ }^{\wedge} p<0.001,{ }^{\wedge \wedge} p<0.01$, and ${ }^{\wedge \wedge \wedge} p<0.05$ versus Scopolamine+Donepezil. (Cont., Control; Scop., Scopolamine; Done., Donepezil; Curc., Curcumin.

of Donepezil in AD rats lowered GLUT3 levels (0.69-fold, $p<0.001)$; however, Curcumin treatment increased the GLUT3 expression (1.16-fold, $p<0.05)$ in $\mathrm{AD}$ rats (Fig. 3h, h') compared to Scopolamine-induced AD rats. In addition, GLUT4 levels in AD rats was significantly decreased (0.87fold, $p<0.001$ ) compared to Saline-treated AD rats. Donepezil and Curcumin treatment separately on AD rats increased GLUT4 levels (1.44-fold, $p<0.001$ and 2.00-fold, $p<0.0010$ ), respectively, compared to Scopolamine-induced rats (Fig. 3i, i').

\section{DISCUSSION}

Hypometabolism of glucose is one of the most important key factors in the AD brain and glucose 
metabolism is linked to insulin signaling pathway. Therefore, impaired insulin signaling may have a pivotal role on impaired cognition such as in AD. Insulin signaling plays important roles in regulation of metabolism of the brain glucose, neuronal development and activities which affect learning and memory [27]. In our studies, we found that the glucose (Fig. 1) and insulin (Fig. 1) levels were decreased in scopolamine-induced brain through the impaired insulin signaling which causes the insulin resistance. These observations indicated that hypometabolism of glucose; hyperinsulinemia and impairment of insulin signaling play roles in the development of $\mathrm{AD}$ $[28,29]$.

In brain, insulin/insulin growth factor (IGF-1) binds to the extracellular $\alpha$-subunit of insulin and IGF-1 receptors (IRs/IGF-1Rs). It induces a conformational change in the tyrosine kinase receptors and leads to the activation of tyrosine kinase that promotes autophosphorylation at several tyrosine residues of the $\beta$-subunit receptor inside the cell that results in the activation of downstream signaling pathway [30].

From previous studies, it was found that lower expression of IRs and IGF-1Rs and IRS expressions progressed with $\mathrm{AD}$ [23-31]. In addition, insulin/ IGF-1 signaling defects predominately decreased PI3K/Akt pathway-mediated GLUTs activation, and their reduced expression in AD brain may result in brain glucose hypometabolism [23]. The decrease of IGF-I levels indicated that the insulin transport into the brain was impaired [32] or deregulation of bloodbrain barrier function was induced by prolonged peripheral hyperinsulinemia $[31,33]$. In this study, we found that the levels of upstream insulin signaling molecules such as IR- $\beta$ (0.72-fold, $p<0.001)$, IGF1 (0.62-fold, $p<0.001)$, IRS- 1 (0.74-fold, $p<0.001$ ), IRS-2 (0.62-fold, $p<0.001)$, p-Akt (Ser473) (0.57fold, $p<0.001)$, and Akt (0.66-fold, $p<0.001)$ were markedly decreased in AD rats compared to Salinetreated control rats (Fig. 3). In addition, IHC study indicated that there was a significant decrease in the localization/and or expression of brain cells immunostained for IRS- 1 and IRS- 2 in AD neurons with signs of neurodegeneration. Furthermore, previous study [34] using Western blot analysis also showed reduced IRS-1 and IRS-2 expressions in the AD brain cortex. In addition, previous study [35] also suggested that IRS-1 is associated with cognitive memory performance as a positive correlation between IRS-1 expression and spatial memory was found in healthy trained animals. Elevated expression of IRS-1 proteins in the brains was observed after spatial training in comparison to control [35]. Importantly, in our studies, we observed reduced expression of IR- $\beta$, IRS- 1 and IRS- 2 in AD rats that impaired insulin signaling cascade in $\mathrm{AD}$ rats.

Akt regulates phosphatidylinositol-3-kinase (PI3K) survival signaling for insulin-like growth factor I (IGF-I) [36]. Akt also has multiple phosphorylation sites and phosphorylation of Serine 473 (Ser473) in Akt allows full activation of Akt [37]. In general, phosphorylation at Ser473 requires the activation of Akt and the levels of phosphorylation at Ser473 denotes the degree of Akt phosphorylation [38]. The activation of Akt plays a beneficial role in neurodegenerative disease such as $\mathrm{AD}$ [39]. In addition, a previous studies [40, 41] had suggested that the activation of Akt prevented neuronal loss in $\mathrm{AD}$ and it was reported that there was a reduced localization of cells immunostained for pAkt (Ser473) in AD brains. In our studies, we observed a reduction in the localization of cells immunostained for Akt and pAkt (Ser473) in AD rats compared to Saline-treated control (Fig. 1). Previous study [37] also showed disruption in Akt signaling is an important contributor in the development of $\mathrm{AD}$. We observed a significant reduction in expression of Akt $(P<0.001)$ and pAkt $($ Ser473) $(p<0.001)$ in $\mathrm{AD}$ rats compared to Saline-treated control rats. Furthermore, a previous study reported that pAkt (Ser473) was significantly reduced in Scopolamineinduced animals [42]. In contrast, another study reported an increased pAkt (Ser473) activity in AD brains [41]. In addition, another study investigated the association of Akt expression and memory performance in Scopolamine-induced animals and they found that Akt expression in the brain was reduced resulting memory impairment [41]. Akt had been found to be an important contributor in Scopolamine-induced memory impairment [42]. In our studies, we also observed a reduced expression of Akt and p-Akt (Ser473) in AD rats that may lead to reduced spatial memory performance. Furthermore, earlier study showed reduced repression of $\mathrm{p}$-Akt in brain/neuron-specific insulin receptor knockout (NIRKO) mice [40]. Interesting in our studies, the lowered expression of p-PI3K (p85) $(p<0.05)$, pAkt (Ser473) $(p<0.001)$, and Akt $(p<0.01)$ in $\mathrm{AD}$ rats were rescued markedly by Curcumintreatment (Fig. 3). It is known that Curcumin has anti-inflammatory property. Neuroinflammation abrogates insulin signaling in the brain [43]. It is possible that Curcumin can prevent inflammation in the $\mathrm{AD}$ rats and thereby improves insulin signaling. 


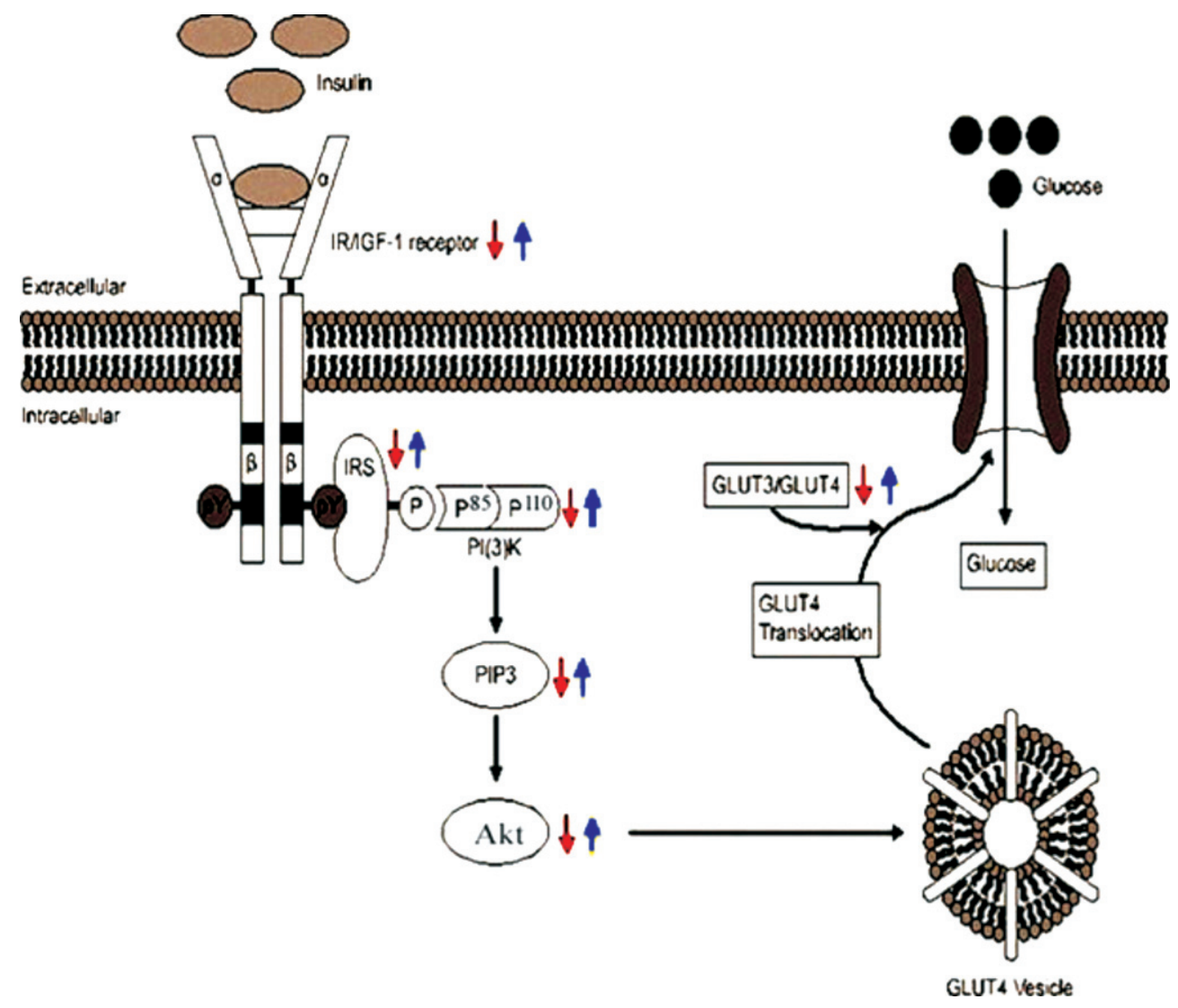

Fig. 4. Schematic representation of insulin signaling and the regulation of glucose uptake in the AD brain (red arrow). In our study, due to the reduced levels of IR/IGF, IRS, PI3K, Akt, and pAkt (Ser473) proteins, insulin signaling was impaired in induced AD rats. In addition, GLUT3 and GLUT4 levels were also decreased in AD rats resulting reduced translocation of glucose transporters into the membrane. Curcumin (blue arrow) rescued lowering of the levels of these signaling proteins and glucose transporters in AD rats.

Interestingly, in this study, we observed a greater effect of Curcumin as opposed to Donepezil, a reference drug used for the treatment of $\mathrm{AD}$, in mediating an active insulin signaling in $\mathrm{AD}$ rats.

GLUT3 is a key neuronal glucose transporter in the brain and it has a higher affinity and more potential $[44,45]$ than GLUT1 for glucose transport $[45$, 46]. Therefore it is believed that a higher GLUT3 expression might improve glucose uptake in the neuron [47]. Previous studies showed that GLUT3 levels were decreased [48-50] in AD-affected brain, suggesting a lowered GLUT3 levels may contribute to AD neurodegeneration. Insulin-sensitive glucose transporter GLUT4 [51-53] has been found in the pituitary and the hypothalamus [54]. Furthermore in cells, there was a slow recycling of GLUT4 between the plasma membrane and the vesicular compartments in the absence of insulin [55]. It was found that insulin stimulates glucose transport by translocation of GLUT4 from the intracellular sites to the plasma membrane [56], although the exact mechanisms are unclear [30, 57]. Furthermore, a study using IHC found that, compared to other GLUTs such as GLUT3 and GLUT4 were predominantly expressed in the brain [58]. Another study reported the association of IRSs/PI3K/Akt pathway with high expression of GLUT3, and GLUT4 translocation as well as their attachment to the plasma membrane [59]. In this study, we observed a reduced localization of cells immunostained with GLUT4 in AD rats compared to Saline-treated control (Fig. 2). A study showed that GLUT4 knockout mice had impaired insulin signaling and developed insulin resistance, however the exact mechanism was unclear [60]. It was also suggested that insulin signaling was impaired due to decreased expression of GLUT3 and GLUT4 [61]. In our study, we also observed that in conjunction with impaired insulin signaling, GLUT3 and GLUT4 levels were reduced in AD rats. Interestingly, Curcumin treatment in $\mathrm{AD}$ rat brain significantly improved 
insulin signaling and rescued the reduced expression of GLUT3 and GLUT4 (Fig. 4). In our study, we observed increased insulin levels in AD rats compared to control rats and Curcumin markedly lowered the insulin levels both in the plasma and in the brain. Hyperinsulinemia was shown to be associated with $\mathrm{AD}$ pathogenesis in humans [62]. In addition, lower levels of glucose were found in $\mathrm{AD}$ patients with significant cognition decline [63]. In our study, glucose levels were decreased in $\mathrm{AD}$ rats compared to control rats and Curcumin significantly rescued the lowered glucose levels in AD rats that may lead to improved cognitive function.

\section{Conclusion}

Taken together in the present study we found downregulation of insulin signaling proteins and major glucose receptors in $\mathrm{AD}$ rats compared to the Saline-treated control rats. Thus our in vivo studies strongly indicate that impaired insulin signaling and altered glucose regulation may contribute to the pathogenesis of AD. These abnormalities observed in Scopolamine-induced rats have been documented in patients with sporadic $\mathrm{AD}$, thereby indicating a possible human relevance of our study. Importantly, in this study, Curcumin has been found to ameliorate the altered insulin signaling in $\mathrm{AD}$ rats, reduce hyperinsulinemia, and improve glucose levels in AD rats. Therefore, Curcumin, a natural compound derived from a plant, may provide a better treatment of $\mathrm{AD}$ patients compared to widely used pharmacological drugs such as Donepezil that may have side effects. Further studies are needed to better understand the mechanism of action of Curcumin in the treatment of $\mathrm{AD}$.

\section{ACKNOWLEDGMENTS}

We thank to Estinnorell Yong, PAPRSB Institute of Health Sciences, Universiti Brunei Darussalam; for her support and contributions to this study. This study was funded by the Universiti Brunei Darussalam/ Brunei Research Council-2(UBD / BRC-2) (Ref: JPKE/DG/83) and Graduate Research Scholarship from UBD (Ref: UBD/GS/1)'2015-16.

\section{CONFLICT OF INTEREST}

The authors have no conflict of interest to report.

\section{REFERENCES}

[1] Alzheimer's Association (2018) Alzheimer's disease facts and figures. Alzheimers Dement 14, 367-429.

[2] Das TK, Mas RW, Das T, Kaneez FS (2015) Potential of glycowithanolides from Withania somnifera (ashwagandha) as therapeutic agents for the treatment of Alzheimer's disease. World J Pharm Res 4, 16-38.

[3] Das TK, Mas RW, Kaneez FS (2014) Oxidative stress gated by Fenton and Haber Weiss reactions and its association with Alzheimer's disease. Arch Neurosci 2, 1-8.

[4] Rocchi A, Pellegrini S, Siciliano G, Murri L (2003) Causative and susceptibility genes for Alzheimer's disease: A review. Brain Res Bull 61, 1-24.

[5] Hoyer S (2004) Causes and consequences of disturbances of cerebral glucose metabolism in sporadic Alzheimer disease: Therapeutic implications. Adv Exp Med Biol 541, 135-152.

[6] Iqbal K, Grundke II (2005) Metabolic/signal transduction hypothesis of Alzheimer's disease and other tauopathies. Acta Neuropathol (Berl) 109, 25-31.

[7] Mattson MP (2004) Pathways towards and away from Alzheimer's disease. Nature 430, 631-639.

[8] Schubert D (2005) Glucose metabolism and Alzheimer's disease. Ageing Res Rev 4, 240-257.

[9] Hartmann J, Kiewert C, Klein J (2010) Neurotransmitters and energy metabolites in amyloid-bearing APP (Swe) xPSEN1de9 Mouse brain. J Pharmacol Exp Ther 332, 364370.

[10] Hooijmans CR, Graven C, Dederen PJ, Tanila H, van Groen T, Kiliaan AJ (2007) Amyloid beta deposition is related to decreased glucose transporter-1 levels and hippocampal atrophy in brains of aged APP/PS1 mice. Brain Res 1181, 93-103.

[11] Cohen E, Dillin A (2008) The insulin paradox: Aging, proteotoxicity and neurodegeneration. Nat Rev Neurosci 9 , 759-767.

[12] Hauptmann S, Scherping I, Dröse S, Brandt U, Schulz KL, Jendrach M, Leuner K, Eckert A, Müller WE (2009) Mitochondrial dysfunction: An early event in Alzheimer pathology accumulates with age in $\mathrm{AD}$ transgenic mice. Neurobiol Aging 30, 1574-1586.

[13] Gerozissis K (2008) Brain insulin, energy and glucose homeostasis: Genes, environment and metabolic pathologies. Eur J Pharmacol 585, 38-49.

[14] Gasparini L, Netzer WJ, Greengard P, Xu H (2002) Does insulin dysfunction play a role in Alzheimer's disease? Trends Pharmacol Sci 23, 288-293.

[15] Steen E, Terry BM, Rivera EJ, Cannon JL, Neely TR, Tavares R, Xu XJ, de la Monte SM (2005) Impaired insulin and insulin-like growth factor expression and signaling mechanisms in Alzheimer's disease-is this type 3 diabetes? J Alzheimers Dis 7, 63-80.

[16] Zhu X, Perry G, Smith MA (2005) Insulin signaling, diabetes mellitus and risk of Alzheimer disease. J Alzheimers Dis 7, 81-84.

[17] Baki L, Neve RL, Shao Z, Shioi J, Georgakopoulos A, Robakis NK (2008) Wild-type but not FAD mutant presenilin-1 prevents neuronal degeneration by promoting phosphatidylinositol 3-kinase neuroprotective signaling. J Neurosci 28, 483-490.

[18] Syed WB, Avninder PS, Manisha T (2012) Supplementation of Convolvulus pluricaulis attenuates scopolamine-induced increased tau and Amyloid precursor protein (A $\beta P P)$ expression in rat brain. Indian J Pharmacol 44, 593-598. 
[19] Bart L, Fabienne A, Anisur R (2013) Animal models of Alzheimer's disease and drug development. Transl Pharmacol 10, 319-327.

[20] Bihaqi SW, Singh AP, Tiwari M (2012) Supplementation of Convolvulus pluricaulis attenuates scopolamine-induced increased tau and amyloid precursor protein (A $\beta P P)$ expression in rat brain. Indian J Pharmacol 44, 593-598.

[21] Haider S, Tabassum S, Perveen T (2016) Scopolamineinduced greater alterations in neurochemical profile and increased oxidative stress demonstrated a better model of dementia: A comparative study. Brain Res Bull 127, 234247.

[22] Anil K, Jyotsna D, Anup S (2011) A review on spice of life curcuma longa turmeric. Int J Appl Biol Pharm 2, 371-379.

[23] Motaghinejad M, Motevalian M, Fatima S, Faraji F, Mozaffari S (2017) The neuroprotective effect of curcumin against nicotine-induced neurotoxicity is mediated by CREB-BDNF signaling pathway. Neurochem Res $\mathbf{4 2}$, 2921-2932.

[24] Guerzoni LP, Nicolas V, Angelova A (2017) In vitro modulation of TrkB receptor signaling upon sequential delivery of curcumin-DHA loaded carriers towards promoting neuronal survival. Pharm Res 34, 492-505.

[25] Angelova A, Angelov B (2017) Dual and multi-drug delivery nanoparticles towards neuronal survival and synaptic repair. Neural Regen Res 12, 886-889.

[26] Rakotoarisoa M, Angelova A (2018) Amphiphilic nanocarrier systems for curcumin delivery in neurodegenerative disorders. Medicines 5, 126.

[27] Enrique B, Esther V, Verónica HC, Juan MRA (2014) Insulin in the brain: Its pathophysiological implications for states related with central insulin resistance, type 2 diabetes and Alzheimer's disease. Front Endocrinol 5, 1-21.

[28] Bryan JN, Craft CS (2017) Insulin resistance and Alzheimer's disease: Bioenergetic linkages. Front Aging Neurosci 9, 1-20.

[29] Berger AL (2016) Insulin resistance and reduced brain glucose metabolism in the aetiology of Alzheimer's disease. $J$ Insulin Resistance 1, 1-7.

[30] Alan RS, Ronald CK (2001) Insulin signalling and the regulation ofglucose and lipid metabolism. Nature 414, 799-806.

[31] Li L, Holscher C (2007) Common pathological processes in Alzheimer disease and type 2 diabetes: A review. Brain Res Rev 56, 384-402.

[32] de la Monte SM (2009) Insulin resistance and Alzheimer's disease. BMB Rep 31, 475-481.

[33] Bosco D, Fava A, Plastine M, Montalcine T, Pujia A (2011) Possible implications of insulin resistance and glucose metabolism in Alzheimer's disease pathogenesis. J Cell Mol Med 15, 1807-1821.

[34] Moloney AM, Griffin RJ, Timmons S, O'Connor R, Ravid R, O'Neill C (2010) Defects in IGF-1 receptor, insulin receptor and IRS-1/2 in Alzheimer's disease indicate possible resistance to IGF-1 and insulin signalling. Neurobiol Aging 31, 224-243.

[35] Zhao WQ, Chen H, Quon MJ, Alkon DL (2004) Insulin and the insulin receptor in experimental models of learning and memory. Eur J Pharmacol 490, 71-81.

[36] Russell JW, Windebank AJ, Schenone A, Feldman EL (1998) Insulin-like growth factor-I prevents apoptosis in neurons after nerve growth factor withdrawal. J Neurobiol 36, $455-467$

[37] Lee HK, Kumar P, Fu Q, Rosen KM, Querfurth HW (2009) The insulin/Akt signaling pathway is targeted by intracellular $\beta$-amyloid. Mol Biol Cell 20, 1533-1544.
[38] Annika R, Nenad B, Inga V, Bengt W, Rivka R, Richard FC (2009) Akt activity in Alzheimer's disease and other neurodegenerative disorders. Neuroreport 15, 955-959.

[39] Cheng B, Martinez AA, Morado J, Scofield V, Roberts JL, Maffi SK (2013) Retinoic acid protects against proteasome inhibition associated cell death in SH-SY5Y cells via the AKT pathway. Neurochem Int 62, 31-42.

[40] Schubert M, Gautam D, Surjo D, Ueki K, Baudler S, Schubert D, Kondo T, Alber J, Galldiks N, Kustermann E (2004) Role for neuronal insulin resistance in neurodegenerative diseases. Proc Natl Acad Sci U S A 101, 3100-3105.

[41] Griffin RJ, Moloney A, Kelliher M, Johnston JA, Ravid R, Dockery P, O'Connor R, O'Neill C (2005) Activation of Akt/PKB, increased phosphorylation of Akt substrates and loss and altered distribution of Akt and PTEN are features of Alzheimer's disease pathology. J Neurochem $\mathbf{9 3}$, 105-117.

[42] Wu Y, Luo X, Liu X, Liu D, Wang X, Guo Z, Wang JZ (2015) Intraperitoneal administration of a novel TAT-BDNF peptide ameliorates cognitive impairments via modulating multiple pathways in two Alzheimer's rodent models. Sci Rep 5, 15032.

[43] Najem D, Bamji-Mirza M, Chang N, Liu QY, Zhang W (2014) Insulin resistance, neuroinflammation, and Alzheimer's disease. Rev Neurosci 25, 509-525.

[44] Hara M, Matsuda Y, Hirai K, Okumura N, Nakagawa H (1989) Characteristics of glucose transport in neuronal cells and astrocytes from rat brain in primary culture. $\mathrm{J} \mathrm{Neu}$ rochem 52, 902-908.

[45] Maher F, Vannucci SJ, Simpson IA (1994) Glucose transporter proteins in brain. FASEB $J \mathbf{8}, 1003-1011$.

[46] Bell GI, Burant CF, Takeda J, Gould GW (1993) Structure and function of mammalian facilitative sugar transporters. J Biol Chem 268, 19161-19164.

[47] Gould GW, Seatter MJ (1997) Introduction to the facilitative glucose transporter family. In Facilitative Glucose Transporters, Gould GW, ed. R.G. Landes Company, Austin, pp. $1-37$.

[48] Kalaria RN, Harik SI (1989) Reduced glucose transporter at the blood-brain barrier and in cerebral cortex in Alzheimer disease. J Neurochem 530, 1083-1088.

[49] Simpson IA, Chundu KR, Davies-Hill T, Honer WG, Davies P (1994) Decreased concentrations of GLUT1 and GLUT3 glucose transporters in the brains of patients with Alzheimer's disease. Ann Neurol 35, 546-551.

[50] Mooradian AD, Chung HC, Shah GN (1997) GLUT-1 expression in the cerebra of patients with Alzheimer's disease. Neurobiol Aging 18, 469-474.

[51] Henriksen EJ, Bourey RE, Rodnick KJ, Koranyi L, Permutt MA, Holloszy JO (1990) Glucose transporter protein content and glucose transport capacity in rat skeletal muscles. Am J Physiol 259, E593-598.

[52] Holman GD, Kozka IJ, Clark AE, Flower CJ, Saltis J, Habberfield AD, Simpson IA, Cushman SW (1990) Cell surface labeling of glucose transporter isoform GLUT4 by bis-mannose photolabel. Correlation with stimulation of glucose transport in rat adipose cells by insulin and phorbol ester. J Biol Chem 265, 18172-18179.

[53] Lawrence JCJ, Piper RC, Robinson LJ, James DE (1992) GLUT4 facilitates insulin stimulation and cAMP-mediated inhibition of glucose transport. Proc Natl Acad Sci U S A 89, 3493-3497.

[54] Brant AM, Jess TJ, Milligan G, Brown CM, Gould GW (1993) Immunological analysis of glucose transporters expressed in different regions of the rat brain and central 
nervous system. Biochem Biophys Res Commun 192, 1297302.

[55] Louise C, Shian-Huey C, Alan RS (2004) Insulin signaling and the regulation of glucose transport. Mol Med 10, 65-71.

[56] Pessin JE, Thurmond DC, Elmendorf JS, Coker KJ, Okada S (1999) Molecular basis of insulin-stimulated GLUT4 vesicle trafficking. Location! Location! Location! J Biol Chem 274, 2593-2596.

[57] Guilherme A, Masahiro E, Joanne MB, Sahana B, Rosanna S, William ET, John L, Michael PC (2000) Perinuclear localization and insulin responsiveness of GLUT4 requires cytoskeletal integrity in 3T3-L1 adipocytes. $J$ Biol Chem 275, 38151-38159.

[58] Choeiri C, Staines W, Messier C (2002) Immunohistochemical localization and quantification of glucose transporters in the mouse brain. Neuroscience 111, 19-34.
[59] Pessin JE, Saltiel AR (2000) Signaling pathways in insulin action: Molecular targets of insulin resistance. J Clin Invest 106, 165-169.

[60] Abel ED, Peroni O, Kim JK, Kim YB, Boss O, Hadro E, Kahn BB (2001) Adipose-selective targeting of the GLUT4 gene impairs insulin action in muscle and liver. Nature $\mathbf{4 0 9}$, 729-733.

[61] Brown GK (2000) Glucose transporters: Structure, function and consequences of deficiency. $J$ Inherit Metab Dis 23, 237-246.

[62] Kim B, Feldman EL (2015) Insulin resistance as a key link for the increased risk of cognitive impairment in the metabolic syndrome. Exp Mol Med 47, 1-10.

[63] Chen Z, Zhong C (2013) Decoding Alzheimer's disease from perturbed cerebral glucose metabolism: Implications for diagnostic and therapeutic strategies. Progr Neurobiol 108, 21-43. 